\title{
Relationship between bone mineral density, its associated physiological factors, and tooth loss in postmenopausal Korean women
}

\author{
Chang-Suk Kim ${ }^{1 \dagger}$, Eun-Kyong Kim ${ }^{2 \dagger}$, Kyeong-Soo Lee ${ }^{3 *}$, Hee-Kyung Lee ${ }^{4}$, Youn-Hee Choi ${ }^{5}$, Tae-Yoon Hwang ${ }^{3}$
} and Jun Sung Moon ${ }^{6}$

\begin{abstract}
Background: Previous studies have proposed a relationship between bone mineral density (BMD) and oral health. However, the relationship between BMD and tooth loss in female individuals is not yet well understood. Therefore, the aim of this study was to assess the association between BMD, including its related physiological factors, and tooth loss among postmenopausal women in Korea.

Methods: A total of 3,992 postmenopausal women aged 50 years or above were selected from the Fourth and Fifth Korea National Health and Nutrition Examination Surveys, which were cross-sectional in design and conducted from 2008 to 2011. The participants' BMD and number of teeth were assessed by radiologists and dentists. Socioeconomic characteristics and female-related physiological factors, including menarche age, duration of menopause, number of pregnancies, age at first child's birth, and duration of oral contraceptive or female hormone use, were surveyed.

Results: Participants who had lower BMD had significantly fewer teeth $(p<0.001)$. Female-related physiological factors, including the duration of menopause, number of pregnancies, age at first child's birth, duration of oral contraceptive or female hormone use, and calcium intake level, showed a significant relationship with the number of teeth. Using multiple regression analysis, BMD, duration of menopause, age at first child's birth, and duration of female hormone use significantly influenced the number of teeth.

Conclusions: BMD and its related physiological factors in female individuals showed a significant relationship with the number of teeth in postmenopausal Korean women, implicating osteoporosis as a risk factor for tooth loss in
\end{abstract} postmenopausal women.

\section{Background}

Osteoporosis is a common metabolic bone disease, often occurring in postmenopausal women, and it is defined as a bone mineral density (BMD) at least 2.5 standard deviations (SDs) below the peak bone mass of a young, healthy, sex- and race-matched reference population, according to the World Health Organization (WHO) [1]. Bone fracture can occur in patients with osteoporosis even as a result of minor trauma to the femur, spine, or wrist [2]. It has been reported that substantial loss of BMD is caused by the

\footnotetext{
* Correspondence: drkslee@ynu.ac.kr

${ }^{\dagger}$ Equal contributors

${ }^{3}$ Department of Preventive Medicine \& Public Health, College of Medicine, Yeungnam University, Daegu, South Korea

Full list of author information is available at the end of the article
}

decrease in estrogen secretion seen in postmenopausal women [3]. Estrogen influences the formation of osteoclast and osteoblast progenitors in bone marrow, and elicits pro-apoptotic effects on osteoclasts and antiapoptotic effects on mature osteoblasts and osteocytes [4]. After menopause, the amount of bone resorption by osteophages is greater than the degree of bone formation by osteoblasts. Furthermore, estrogen deficiency accelerates bone turnover, thereby interfering with the absorption of calcium by the intestines and resulting in an increase in the excretion of calcium [5].

Menopause in women has also been reported to affect oral health [6]. For example, estrogen deficiency may reduce the healing ability of mucosal wounds and may contribute to a reduced salivary flow rate, which may in 
turn affect the periodontal tissues, including alveolar bone, in cases of poor oral hygiene [7, 8]. Severe alveolar bone loss is a well-known cause of tooth loss [9].

Many studies have reported an association between osteoporosis and BMD in the mandible [10-12]. Ward and Manson reported that osteoporosis did not cause periodontitis but increased the rate of BMD reduction [13]. Hunziker et al. reported that a decrease in estrogen secretion may lead to osteoporosis and BMD reduction in the mandible when periodontitis is already present [14]. In addition, Tezal et al. reported that skeletal BMD was associated with periodontal tissues, such as alveolar bone, and clinical attachment [15].

In light of the results of previous studies, it is necessary to assess the relationship between BMD and oral health in postmenopausal women, because an increasing number of osteoporosis patients are being seen as a result of our aging population. Cooper et al. suggested that the number of hip fractures occurring because of osteoporosis would rise from 1.66 million in 1990 to 6.26 million by 2050, and that approximately $50 \%$ of hip fractures would occur in Asia [16]. According to the Fourth Korea National Health and Nutrition Examination Survey (KNHANES), $39.1 \%$ of Korean women aged 50 years and older were diagnosed with osteoporosis in Korea [17]. In addition, the BMD of Korean women aged 20-49 years was lower when compared with that of women in the US and Japan [18]. A previous study reported on the relationship between periodontal disease, tooth loss, and BMD in a Korean population; however, the study had certain limitations, including possible bias and the inability of the study population to adequately represent the general population [19]. Therefore, we analyzed data from a nationally representative survey to overcome such limitations.

The purpose of this study was to assess the relationship between BMD and tooth loss, and to identify the determinant female-related physiological factors among postmenopausal women using data from the KNHANES.

\section{Methods}

\section{Study participants}

This study analyzed data from the second and third years of the Fourth KNHANES (2008-2009) and the first and second years of the Fifth KNHANES (20102011). Data from KNHANES was publicly available and released from Korea Center for Disease Control and Prevention. The KNHANES, a nationally representative, complex, stratified, and multistage sample survey, was conducted by the Ministry of Health and Welfare, Korea. A total of 3,992 postmenopausal women aged 50 years or older who underwent both a BMD test and oral health examination were included. The KNHANES protocol was approved by the institutional review board of the Korea Centers for Disease Control and Prevention (IRB No = 2008-04EXP-01-C, 2009-01CON-03-2C, 2010-02CON-21-C, 2011-02CON-06-C).

\section{Assessment of BMD}

BMD was assessed by trained radiologists by evaluating the lumbar spine and left femoral neck using dualenergy X-ray absorptiometry with a fan-beam densitometer (Discovery-W, Hologic, Inc., USA). If the left femur could not be adequately measured, e.g., because of surgery or fracture, then the right femur was measured. The T-score, which is a widely used measure for osteoporosis screening, was calculated using the maximal BMD data from Japanese criteria [20]. In accordance with WHO standards, a T-score above -1.0 was considered normal, between -1.0 and -2.5 was considered osteopenia, and less than -2.5 was diagnosed as osteoporosis [1].

\section{Assessment of tooth loss}

For assessment of tooth loss, trained dentists counted the number of teeth, which was defined as the number of permanent teeth excluding the third molars at the time of the oral examination.

\section{Questionnaires}

Each participant completed a questionnaire consisting of six items on socioeconomic characteristics (age, income, education level, body mass index (BMI), family history of osteoporosis, calcium intake, and BMD) and six items on physiological factors (menarche age, duration of menopause, number of pregnancies, age at first child's birth, and duration of oral contraceptive and female hormone use). Income was classified into four groups according to quartiles of income level. BMI was calculated by dividing the participant's weight by the square of their height $\left(\mathrm{kg} / \mathrm{m}^{2}\right)$, and this was classified into three groups (underweight: $<18.5$, normal: $\geq 18.5$ and $<25$, and obese: $\geq 25$ ). Calcium intake was divided into four groups according to quartiles of calcium intake level.

\section{Statistical analysis}

Sampling weights were applied to compensate for sample design and non-response, and integrating weights were applied for the analysis of combined data from the Fourth and Fifth KNHANES [21]. To describe the socioeconomic and health-related characteristics of the participants, data were categorized and reported as weighted percentages. For the comparison of the difference in the number of teeth according to BMD and female-related physiological factors, the analysis was performed using one-way analysis of variance. Multiple regression analysis was performed to determine the association between $\mathrm{BMD}$, its female-related physiological factors, and the 
number of teeth. To test interaction by age, a general linear model was used. The level of statistical significance was set at a two-tailed $p$-value of 0.05 . All statistical analyses were performed using SAS version 9.1.2 (SAS Institute, Inc., Cary, NC, USA).

\section{Results}

The distribution of socioeconomic and health-related characteristics of the participants is shown in Table 1 . The mean age of the participants was $64 \pm 0.2$ years (range: 50-95 years). Approximately half of the participants had osteopenia (48.99\%) and osteoporosis was seen in $37.60 \%$ of the participants. The level of subjective monthly income was almost equally distributed. The education level of the majority of participants was less than elementary school $(n=2,723 ; 65 \%)$, and based on BMI, most participants were classified as either normal $(n=2,394 ; 61 \%)$ or obese $(n=1,489 ; 37 \%)$.

The association between female-related physiological factors, BMD, and the number of teeth for all participants is shown in Table 2. Overall, participants with a longer duration of menopause, greater number of pregnancies, earlier age at first child's birth, not taking female hormones, and with a reduced intake of calcium had significantly fewer teeth $(p<0.01)$. In the 50 - to 64 -

Table 1 Socioeconomic characteristics and health-related characteristics of participants

\begin{tabular}{|c|c|c|}
\hline Variable & Category & $N\left(\%{ }^{a}\right)$ \\
\hline \multirow[t]{3}{*}{ Age(years) $)^{b}$} & $50-59$ & $1,301(40)$ \\
\hline & $60-69$ & $1,433(31)$ \\
\hline & $70+$ & $1,258(29)$ \\
\hline \multirow[t]{4}{*}{ Subjective monthly income } & Low & 974(24) \\
\hline & Middle-low & $1,011(25)$ \\
\hline & Middle-high & $996(26)$ \\
\hline & High & $953(25)$ \\
\hline \multirow[t]{4}{*}{ Education level } & Less than elementary school & $2,723(65)$ \\
\hline & Less than middle school & $543(15)$ \\
\hline & Less than high school & $545(15)$ \\
\hline & Graduate of college or higher & $164(4.7)$ \\
\hline \multirow[t]{3}{*}{ Body mass index } & Underweight & $102(2.2)$ \\
\hline & Normal & 2,394(61) \\
\hline & Obesity & $1,489(37)$ \\
\hline \multirow{2}{*}{$\begin{array}{l}\text { Family history of parents } \\
\text { with osteoporosis }\end{array}$} & Yes & $646(16)$ \\
\hline & No & $3,320(84)$ \\
\hline \multirow[t]{3}{*}{ Bone mineral density } & Normal & 490(13) \\
\hline & Osteopenia & $1,910(49)$ \\
\hline & Osteoporosis & $1,592(38)$ \\
\hline Total & & $3,992(100)$ \\
\hline
\end{tabular}

year-old group, the duration of female hormone use showed an association with the number of teeth. However, for participants aged 65 years or above, the age at first child's birth, duration of female hormone use, and calcium intake were associated with the number of teeth $(p<0.05)$.

The relationship between BMD and the number of teeth is shown in Table 3. Overall, participants with a greater tendency towards osteoporosis displayed significantly fewer teeth $(p<0.001)$.

Multiple regression analysis was performed to determine factors affecting the number of teeth. Variables including age, high income, graduating from middle school or lower and graduating from university or higher in educational level, presence of osteoporosis, duration of menopause, age at first child's birth, and duration of female hormone use showed significant associations with the number of teeth. As age increased by 1 year, the number of teeth decreased by 0.31 . Participants who had osteoporosis had 1.54 fewer teeth when compared with healthy participants. As the duration of menopause increased by 1 year, the number of teeth decreased by 0.16 . In addition, as the age at the first child's birth and the duration of female hormone use increased by 1 year, the number of teeth increased by 0.19 and 0.03 , respectively (Table 4).

\section{Discussion}

In this study, we observed a significant relationship among postmenopausal women between BMD, its related determinant factors, and the number of teeth. Using multiple regression analysis we observed that female-related physiological factors, including the duration of menopause, age at first child's birth, and duration of female hormone use, were associated with the number of teeth. To the best of our knowledge, this is the first study assessing the relationship between BMD, its related factors, and tooth loss among postmenopausal women in Korea using a national survey (KNHANES).

In our study, the association between BMD and the number of teeth was statistically significant. BMD and the number of teeth were known to be significantly affected by an increase in age. As such, we stratified and analyzed age to control it as a variable [22]. Overall, participants with a greater tendency towards osteoporosis displayed significantly fewer teeth $(p<0.001)$, which coincided with the results of a previous study by Tak et al. [19]. Based on this result, it is reasonable to consider that female-related physiological factors associated with osteoporosis are significantly related with the number of teeth.

Gur et al. reported that BMD decreased with an increase in the 'period of menopause' [23]. These results could be inferred from the relationship between estrogen 
Table 2 Association between female related physiological factors and the number of teeth

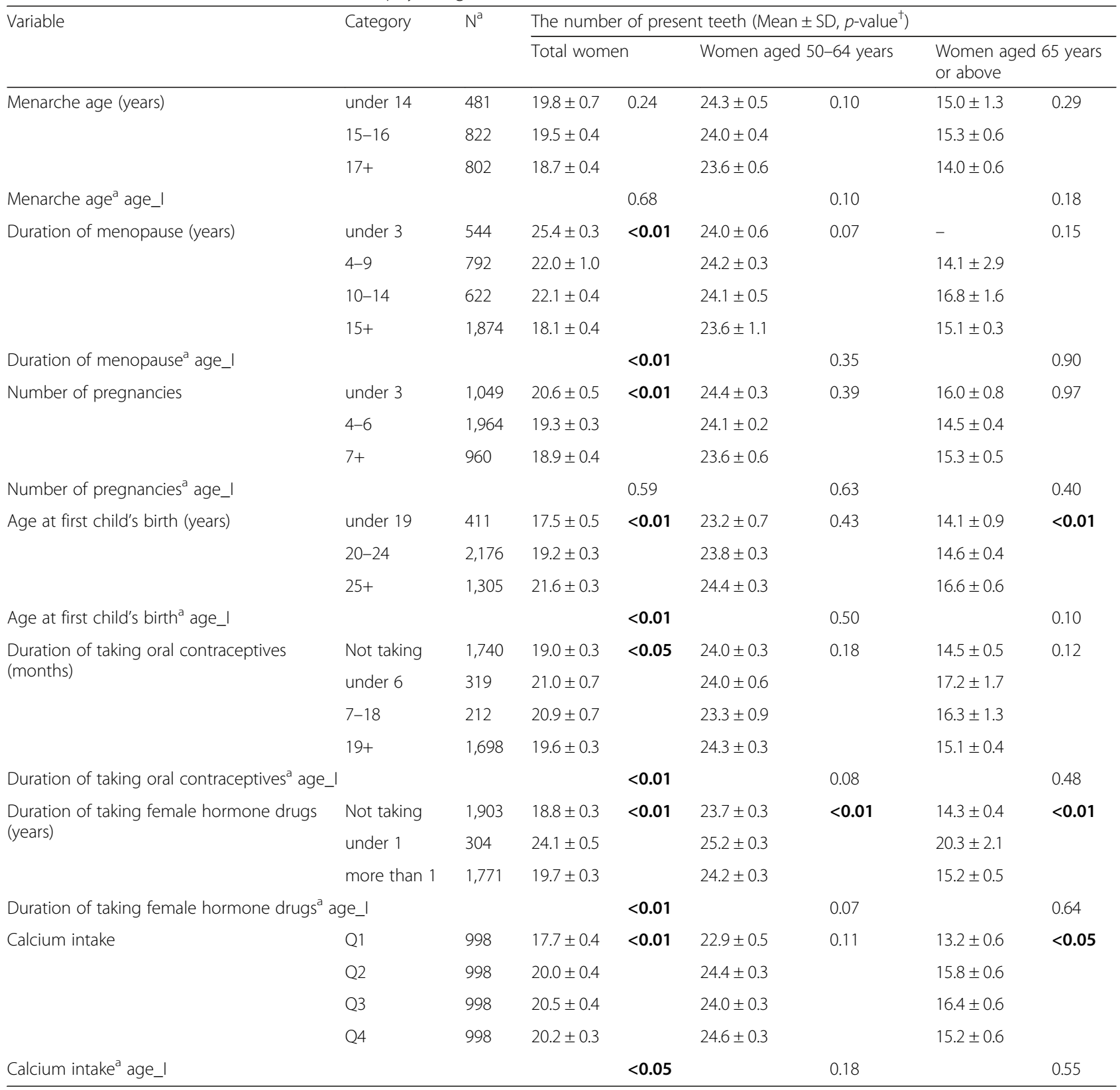

${ }^{a}$ Exclude missing values

${ }^{\dagger} p$-values obtained from oneway ANOVA using general linear model for complex survey design

age_l: to test interaction by age, participants were categorized into two age groups (50-64 years and $\geq 65$ years

and BMD. Notelvitz reported that osteoblasts indirectly regulate the resorption activity of osteoclasts under the influence of estrogen [24]. Simm et al. reported that estrogen produces growth factors and contributes to bone formation [25]. Based on these findings, hormone replacement therapy has been used in the treatment of osteoporosis [26, 27]. The results of our study showed that participants taking female hormones for more than 1 year had a greater number of teeth present than those not taking female hormones. Again, this result can be inferred from the decreased estrogen secretion occurring in the latter group. According to Hunziker et al., a decrease in estrogen secretion may lead to BMD loss in the mandible in patients with periodontitis [14]. Tezal et al. reported that systemic bone resorption affected periodontal tissues and that a common pathway for destruction exists between systemic and periodontal bone [15]. Thus, this hypothesis supported our findings. 
Table 3 Association between bone mineral density and the number of teeth

\begin{tabular}{|c|c|c|c|c|c|c|c|c|c|c|c|c|}
\hline \multirow[t]{3}{*}{ Variables } & \multirow{2}{*}{\multicolumn{4}{|c|}{ Total }} & \multicolumn{8}{|c|}{ Number of teeth } \\
\hline & & & & & \multicolumn{4}{|c|}{ Age $50-64$ years } & \multicolumn{4}{|c|}{ Age $\geq 65$ years } \\
\hline & Total $^{a}$ & $\mathrm{~N}$ & Mean \pm SD & $p$-value ${ }^{\dagger}$ & Total $^{\mathrm{a}}$ & $\mathrm{N}$ & Mean \pm SD & $p$-value ${ }^{\dagger}$ & Total $^{a}$ & $\mathrm{~N}$ & Mean \pm SD & $p$-value ${ }^{\dagger}$ \\
\hline Normal & 3,410 & 436 & $23.4 \pm 0.6$ & $<0.01$ & 1,767 & 375 & $25.2 \pm 0.3$ & 0.06 & 1,643 & 61 & $20.4 \pm 1.7$ & 0.06 \\
\hline Osteopenia & & 1,656 & $20.7 \pm 0.3$ & & & 1,038 & $23.8 \pm 0.3$ & & & 618 & $16.4 \pm 0.5$ & \\
\hline Osteoporosis & & 1,318 & $18.2 \pm 0.3$ & & & 354 & $24.0 \pm 0.4$ & & & 964 & $13.9 \pm 0.4$ & \\
\hline $\mathrm{BMD}^{\mathrm{a}}$ age_I & & & & $<0.01$ & & & & 0.39 & & & & 0.08 \\
\hline
\end{tabular}

${ }^{\text {aExclude missing values }}$

${ }^{\dagger} p$-values obtained from oneway ANOVA using general linear model for complex survey design

age_l: to test interaction by age, participants were categorized into two age groups (50-64 years and $\geq 65$ years).

However, unexpectedly, participants taking female hormones for more than 1 year showed a lower number of teeth present than those taking female hormones for less than 1 year. This may have resulted from the relatively small number and young age of the participants who took female hormones for less than 1 year. This can be confirmed by the fact that out of 304 participants who took female hormones for less than 1 year, 228 and 76 participants were aged 50-64 years and 65 years or above, respectively.

Nevertheless, because of the limitations of crosssectional surveys, it may be difficult to determine a causal relationship. Therefore, prospective studies are required in the future. However, the major advantages of our study were that the participants represented the general female population of Korea, and that more reliable examination results were obtained because of the use of trained examiners, including dentists and radiologists trained by a government organization.

The results of this study showed that participants with a greater tendency towards osteoporosis had fewer teeth present. Therefore, if future prospective studies investigating the relationship between these variables are conducted, we suggest that medical doctors and dentists work together in providing information to promote overall health in postmenopausal women.

Table 4 Multiple regression analysis of factors influencing on the number of teeth

\begin{tabular}{|c|c|c|c|c|}
\hline \multirow[t]{2}{*}{ Variable } & \multicolumn{2}{|l|}{ Crude } & \multicolumn{2}{|l|}{ Adjusted } \\
\hline & Beta Coefficients (Standard error) & $p$-value & Beta Coefficients (Standard error) & $p$-value \\
\hline \multicolumn{5}{|l|}{ Bone mineral density (ref. = normal) } \\
\hline Osteopenia & $-3.23(0.38)$ & $<0.01$ & $-0.70(0.42)$ & 0.10 \\
\hline Osteoporosis & $-8.88(0.45)$ & $<0.01$ & $-1.54(0.60)$ & $<0.05$ \\
\hline Menarche age (years) & $-0.88(0.14)$ & $<0.01$ & $-0.02(0.13)$ & 0.90 \\
\hline Duration of menopause (years) & $-0.45(0.02)$ & $<0.01$ & $-0.16(0.05)$ & $<0.01$ \\
\hline Number of pregnancies & $-0.84(0.09)$ & $<0.01$ & $0.14(0.12)$ & 0.21 \\
\hline Age at first child's birth (years) & $0.69(0.06)$ & $<0.01$ & $0.19(0.07)$ & $<0.01$ \\
\hline Duration of taking oral contraceptives (months) & $0.000(0.000)$ & 0.67 & $0.02(0.02)$ & 0.15 \\
\hline Duration of taking female hormone drugs (years) & $-0.000(0.000)$ & 0.20 & $0.03(0.007)$ & $<0.01$ \\
\hline Calcium intake & $0.003(0.001)$ & $<0.01$ & $0.001(0.000)$ & $<0.01$ \\
\hline Age (years) & $-0.57(0.02)$ & $<0.01$ & $-0.31(0.06)$ & $<0.01$ \\
\hline \multicolumn{5}{|l|}{ Subjective monthly income (ref. = low) } \\
\hline Middle-low & $0.91(0.50)$ & 0.07 & $1.29(0.64)$ & 0.05 \\
\hline Middle-high & $0.77(0.56)$ & 0.17 & $0.87(0.66)$ & 0.18 \\
\hline High & $2.25(0.51)$ & $<0.01$ & $2.12(0.63)$ & $<0.01$ \\
\hline \multicolumn{5}{|l|}{ Education level (ref. = less than elementary school) } \\
\hline Less than middle school & $6.29(0.44)$ & $<0.01$ & $1.58(0.57)$ & $<0.01$ \\
\hline Less than high school & $6.82(0.46)$ & $<0.01$ & $1.42(0.68)$ & $<0.05$ \\
\hline \multirow[t]{2}{*}{ Graduate of college or higher } & $8.31(0.45)$ & $<0.01$ & $2.54(0.75)$ & $<0.01$ \\
\hline & & & Adjusted $R^{2}=0.34$ & \\
\hline
\end{tabular}




\section{Conclusions}

The findings of this study showed that postmenopausal women with low BMD had a significantly lower number of teeth when compared with healthy women. Furthermore, BMD-related physiological factors in postmenopausal female individuals showed an association with the number of teeth, implicating osteoporosis as a risk factor for tooth loss in postmenopausal women.

\section{Abbreviations}

BMD: Bone mineral density; BMI: Body mass index; KNHANES: Korea National Health and Nutrition Examination Survey; SD: Standard deviation; WHO: World Health Organization.

\section{Competing interests}

The authors declare that they have no competing interests.

\section{Authors' contributions}

KSL and C-SK designed this study and drafted the original manuscript. C-SK collected data and performed data analyses. $\mathrm{H}-\mathrm{KL}$ and $\mathrm{Y}-\mathrm{HC}$ interpreted data. T-YH and JSM provided critical comments on the original manuscript and contributed to development of the final draft. E-KK wrote the final manuscript. All authors read and approved the final manuscript.

\section{Author details}

${ }^{1}$ Department of Dental Hygiene, Ulsan College, Ulsan, South Korea. ${ }^{2}$ Department of Dental Hygiene, College of Science \& Technology, Kyungpook National University, Sangju, South of Korea. ${ }^{3}$ Department of Preventive Medicine \& Public Health, College of Medicine, Yeungnam University, Daegu, South Korea. ${ }^{4}$ Department of Dentistry, Yeungnam University, Daegu, South Korea. ${ }^{5}$ Department of Preventive Dentistry, School of Dentistry, Kyungpook National University, Daegu, South Korea. ${ }^{6}$ Department of Internal Medicine, Yeungnam University, Daegu, South Korea.

Received: 30 December 2014 Accepted: 4 August 2015

Published online: 26 August 2015

\section{References}

1. World Health Organization. WHO technical report series 843. Assessment of fracture risk and its application to screening for postmenopausal osteoporosis. Geneva: World Health Organization; 1994.

2. Burge R, Dawson-Hughes B, Solomon DH, Wong JB, King A, Tosteson A. Incidence and economic burden of osteoporosis-related fractures in the United States, 2005-2025. J Bone Miner Res. 2007;22:465-75.

3. Bone HG, Greenspan SL, McKeever C, Bell N, Davidson M, Downs RW. Alendronate and estrogen effects in postmenopausal women with low bone mineral density. Alendronate/Estrogen Study Group. J Clin Endocrinol Metab. 2000;85:720-6.

4. Imai Y, Youn MY, Kondoh S, Nakamura T, Kouzmenko A, Matsumoto T, et al. Estrogens maintain bone mass by regulating expression of genes controlling function and life span in mature osteoclasts. Ann N Y Acad Sci. 2009;1173 Suppl 1:E31-39.

5. Zhao R. Immune regulation of osteoclast function in postmenopausalosteoporosis: a critical interdisciplinary perspective. Int J Med Sci. 2012;9:825-32.

6. Yalcin F, Gurgan S, Gul G. Oral health in postmenopausal Turkish women. Oral Health Prev Dent. 2006:4:227-33.

7. Buencamino MC, Palomo L, Thacker HL. How menopause affects oral health, and what we can do about it. Cleve Clin J Med. 2009;76:467-75.

8. D R M, G K, K J, D D, T V S, Dinesh P. Evaluation of Salivary Flow Rate, pH and Buffer in Pre, Post \& Post Menopausal Women on HRT. J Clin Diagn Res. 2014;8:233-6.

9. Tezal M, Wactawski-Wende J, Gross SG, Dmochowski J, Genco RJ. Periodontal disease and the incidence of tooth loss in postmenopausal women. J Periodontol. 2005;76:1123-8.

10. Cakur B, Dagistan S, Sahin A, Harorli A, Yilmaz A. Reliability of mandibular cortical index and mandibular bone mineral density in the detection of osteoporotic women. Dentomaxillofac Radiol. 2009;38:255-61.
11. Makker A, Singh MM, Mishra G, Singh BP, Jain GK, Jadhav S. Relationship between bone turnover biomarkers, mandibular bone mineral density, and systemic skeletal bone mineral density in premenopausal and postmenopausal Indian women. Menopause. 2012;19:642-9.

12. Horner K, Allen P, Graham J, Jacobs R, Boonen S, Pavitt S, et al. The relationship between the OSTEODENT index and hip fracture risk assessment using FRAX. Oral Surg Oral Med Oral Pathol Oral Radiol Endod. 2010;110:243-9.

13. Ward VJ, Manson JD. Alveolar bone loss in periodontal disease and metacarpal index. J Clin Periodontol. 1973;44:763-9.

14. Hunziker J, Wronski TJ, Miller SC. Mandibular bone formation rates in aged ovariectiomized rats treated with anti-resorptive agents alone and in combination with intermittent parathyroid hormone. J Dent Res. 2000;79:1431-8.

15. Tezal M, Wactawski-Wende J, Grossi SG, Ho AW, Dunford R, Genco RJ. The relationship between bone mineral density and periodontitis in postmenopausal women. J Periodontol. 2000;71:1492-8.

16. Cooper C, Campion G, Melton 3rd $\sqcup$. Hip fractures in the elderly: a world-wide projection. Osteoporos Int. 1992;2:28528-9.

17. Kim KH, Lee K, Ko YJ, Kim SJ, Oh SI, Durrance DY, et al. Prevalence, awareness, and treatment of osteoporosis among Korean women: The Fourth Korea National Health and Nutrition Examination Survey. Bone. 2012;50:1039-47.

18. Park EJ, Joo IW, Jang MJ, Kim YT, Oh K, Oh HJ. Prevalence of osteoporosis in the Korean population based on Korea National Health and Nutrition Examination Survey (KNHANES), 2008-2011. Yonsei Med J. 2014;55:1049-57.

19. Tak IH, Shin MH, Kweon SS, Nam HS, Cauley JA, Kim OJ, et al. The association between periodontal disease, tooth loss and bone mineral density in a Korean population. J Clin Periodontol. 2014;41:1139-44.

20. Orimo H, Hayashi Y, Fukunaga M, Sone T, Fujiwara S, Shiraki M, et al. Osteoporosis Diagnostic Criteria Review Committee: Japanese Society for Bone and Mineral Research. Diagnostic criteria for primary osteoporosis: year 2000 revision. J Bone Miner Metab. 2001;19:331-7.

21. The Korean Association for Survey Research. Weighting Adjustment of the 4th KNHANES (2007 2009) and Sampling Design of the 5th (2010 2012) KNANES, Online information available at https://knhanes.cdc.go.kr/knhanes/ index.do [accessed 22 February 2011].

22. Lee JK, Kim EJ, Seok MH, Kim EY, Hwang RI. Factors influencing osteoporosis. J Korean Acad Community Health Nurs. 2003;14:253-62.

23. Gur A, Nas K, Kayhan O, Atay MB, Akyuz G, Sindal D, et al. The relation between tooth loss and bone mass in postmenopausal osteoporotic women in Turkey: a multicenter study. J Bone Miner Metab. 2003;21:43-7.

24. Notelvitz M. Osteoporosis: screening, prevention and management. Fertil Steril. 1993;59:707-25.

25. Simm PJ, Bajpai A, Russo VC, Werther GA. Estrogens and growth. Pediatr Endocrinol Rev. 2008:6:32-41.

26. Waaseth M, Bakken K, Dumeaux V, Olsen KS, Rylander C, Figenschau Y, et al. Hormone replacement therapy use and plasma levels of sex hormones in the Norwegian Women and Cancer postgenome cohort - a cross-sectional analysis. BMC Womens Health. 2008;8:1.

27. Du Y, Dören M, Melchert HU, Scheidt-Nave C, Knopf H. Differences in menopausal hormone therapy use among women in Germany between 1998 and 2003. BMC Womens Health. 2007;7:19. 